\title{
Investigation on melt pool characterization in laser surface remelting using simulation approach and VIS-SWIR imaging
}

\author{
by P. Koruba*, P. Jurewicz* and J. Reiner* \\ *Wroclaw Univ. of Technology, 50-371, Lukasiewicz Str., Wroclaw, Poland, piotr.koruba@pwr.edu.pl
}

\begin{abstract}
The characterization of melt pool in laser surface remelting gives useable data for prediction of the process result as well as it may constitute a basis for the study of more advanced processes such as laser metal deposition. In this study the analysis of melt pool was performed using two approaches: development of simulation model and coaxial measurement within VIS-SWIR bands. Moreover the simulation model contains multispectral radiative heat transfer calculation, which results can be directly compared with images from the cameras. As a result an evaluation of utilized spectral bands in case of laser material processing monitoring is proposed.
\end{abstract}

\section{Introduction}

Laser surface remelting (LSR) is a technology used widely in laser material processing for enhancing properties of surface layer of the components [1]. It is also a simplification of the selective laser melting (SLM) or Laser Metal Deposition (LMD) processes, that are considered as common additive manufacturing technologies. Currently such processes can be monitored using various non-contact sensors, where the output of the measurement is dimensionless intensity [2] or temperature [3]. On the other hand the simulation tools enable the development of the multi-physical model of the process, in which one can obtain the information about phase change [4], temperature field [5] and emission from the melt pool [6]. However, there is lack of reports treating about the comparison between modelling results and experimental data in case of melt pool characterization (shape and dimensions) based on its emitted radiation.

In this study a comparison of melt pool simulation model and experimental data obtained with spectrometers and cameras from ViS to SWIR spectra was proposed in order to characterize the melt pool and the heat affected zone behind it using different acquisition settings. Moreover the usage of two spectral bands allowed evaluation of the utilised bands for monitoring of the melt pool in laser material processing.

\section{Materials and methods}

LSR process may be applied to a great variety of materials, yet the material chosen for this study is AISI $316 \mathrm{~L}$ steel that can be regarded as a representative of stainless steels group. Therefore its thermal and radiative properties are widely available in literature. In case of simulation model all of the material properties were temperature dependent.

\subsection{Description of LSR process model development}

The model of LSR process was developed within Comsol Multiphysics software due to the modular structure of the model. It contains the time dependant study including heat transfer phenomena such as deposited beam heat source, conductivity of the substrate, phase change from solid to liquid state as well as fluid dynamics in the formed melt pool and multispectral radiation analysis.

\subsection{Experimental setup for LSR process monitoring}

The LSR process was conducted using high power diode laser Laseline LDF 4000-30 (HPDL) and 3-axis CNC portal system enabling coaxial measurement of the melt pool by mounting the sensors to auxiliary optical path of the laser head. For the characterization of melt pool following sensors were used:

- $\quad$ spectrometers (Ocean Optics USC2000+ with spectral range 200-850 nm, Ocean Optics NIRQUEST 512-2.5 with spectral range $900-2500 \mathrm{~nm}$ ),

- $\quad$ cameras (VIS - Photonfocus MV1-D1312-160-CL12, SWIR - Allied Vision Goldeye G-008).

In case of all the sensors a band-stop filter was used in order to eliminate the HPDL laser irradiation during measurement.

\section{Results from LSR process simulation}

The quantities describing the melt pool in LSR process such as temperature, liquid phase indicator and radiosity in VIS and SWIR band were derived using developed multiphysical LSR process model and shown in fig. 1. Moreover the profiles along the direction of remelting for each of the quantity were compared in order to show the possible range of temperature and molten metal monitoring for selected spectral bands. From the obtained plots one can state that both VIS 
and SWIR bands are suitable for characterization of the melt pool yet the SWIR band gives an opportunity to describe the heat affected zone behind the melt pool.
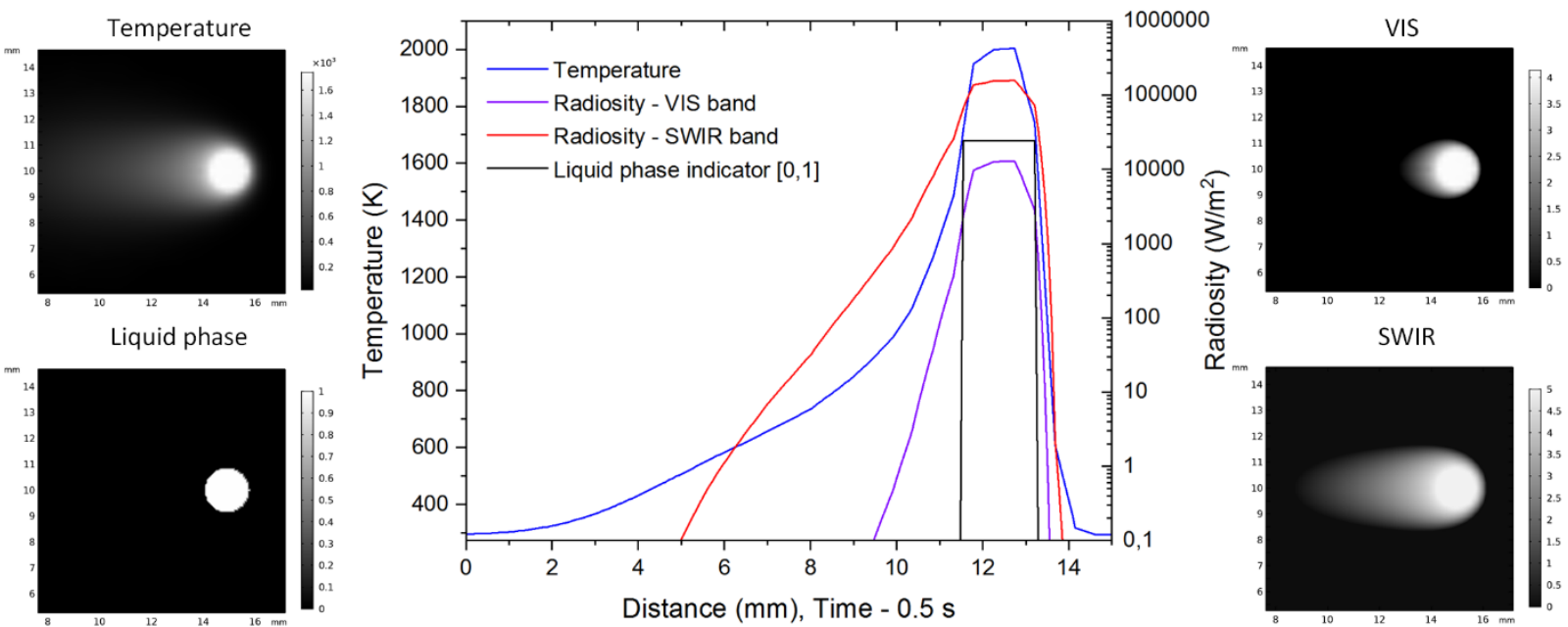

Fig. 1. Comparison of quantities describing melt pool from multiphysical simulation of LSR process

\section{Preliminary experimental results}

As a first step of the experimental study, the measurement with spectrometers was carried out. The result shows that in case of VIS band the highest intensity from the melt pool was obtained between 0.5 and $0.8 \mu \mathrm{m}$, whereas in SWIR band the intensity peak occurs around $1.7 \mu \mathrm{m}$ (fig. 2a), therefore the selection of VIS and SWIR camera can be recognized as correct. The preliminary results obtained in measurements with VIS and SWIR camera were shown in Fig. $2 b, c$. The images indicate that the extended selection of acquisition parameters should be performed for more complete imaging of simulation results.

a)

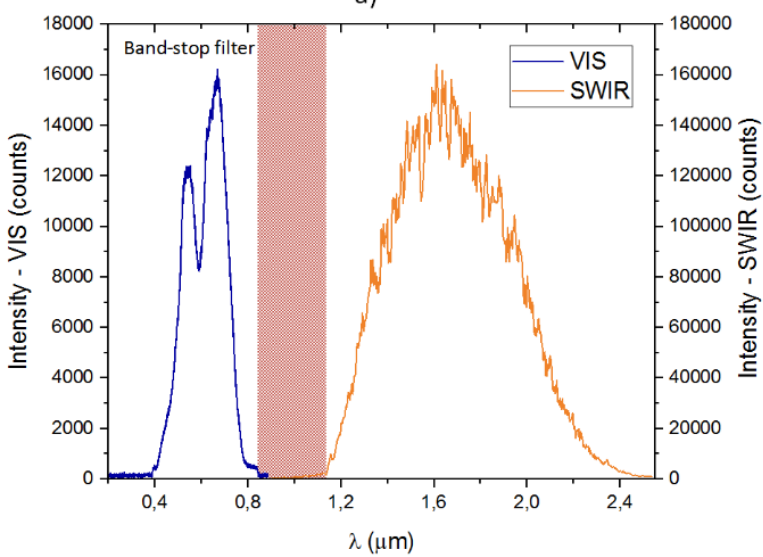

b)

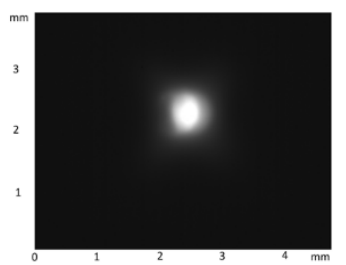

c)

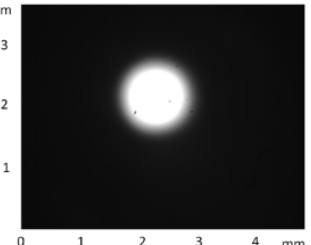

Fig. 2. Results obtained with VIS-SWIR spectrometers (a), VIS camera (b) and SWIR camera (c)

\section{REFERENCES}

[1] Brytan Z., Bonek M., Dobrzanski L. et al., The Laser Surface Remelting of Austenitic Stainless Steel, Materials Science Forum, vol. 654-656 (2010) pp 2511-2514, 2010.

[2] Lough C.S. Wang X., Smith C.C. et al., Use of SWIR Imaging to Monitor Layer-to-Layer Part Quality during SLM of 304L Stainless Steel, Solid Freeform Fabrication 2018: Proceedings of the 29th Annual International Solid Freeform Fabrication Symposium - An Additive Manufacturing Conference, Austin (USA), 2018.

[3] Coniglio N., Mathieu A., Aubreton O. et al., Weld pool surface temperature measurement from polarization state of thermal emission, Quantitative InfraRed Thermography Journal. - Vol. 13, no 1 (2016), pp. 83-93, 2016.

[4] Leitz K.-H., Singer P., Plankensteiner A., Tabernig B., Kestler H, Sigl L.S., Multi-physical simulation of selective laser melting, Metal Powder Report, vol. 72, no. 5 (2017), pp. 331-338, 2017.

[5] Hoadley A., Rappaz M., Zimmermann M., Heat-Flow Simulation of Laser Remelting with Experimental Validation, Metallurgical Transactions B vol. 22 (1991), pp. 101-109, 1991.

[6] Gusarov A.V., Yadroitsev I., Bertrand Ph. et al., Model of Radiation and Heat Transfer in Laser-Powder Interaction Zone at Selective Laser Melting, Journal of Heat Transfer, vol. 131, no. 7(2009), 72101-1, 2009. 